
\title{
25 Research Soure \\ Synthesis and characterization of black TiO2/graphene composites with enhanced photocatalysis
}

\section{Zhaoqing Li}

Huazhong University of Science and Technology

\section{Zhufeng Liu}

Huazhong University of Science and Technology

\section{Xiao Yang}

Huazhong University of Science and Technology

\section{Peng Chen}

Huazhong University of Science and Technology

\section{Lei Yang}

Huazhong University of Science and Technology

\section{Rongzhen Liu}

Huazhong University of Science and Technology

Chunze Yan ( $\nabla$ c_yan@hust.edu.cn )

Huazhong University of Science and Technology

\section{Research Article}

Keywords: Titanium dioxide, Graphene, Photocatalysis, Hydrogenation, Ti3+ self-doping

Posted Date: December 16th, 2020

DOI: https://doi.org/10.21203/rs.3.rs-128642/v1

License: (c) (1) This work is licensed under a Creative Commons Attribution 4.0 International License. Read Full License 


\section{Abstract}

According to the composite design, a series of black $\mathrm{TiO}_{2} /$ graphene composites were synthesized to improve its photocatalytic activity. $\mathrm{TiO}_{2}$ is generated in situ on the surface of graphene by a facile sol-gel method. The combination of graphene and $\mathrm{TiO}_{2}$ was beneficial for eliminating the opportunity of photogenerated electron-hole recombination due to the excellent conductivity of graphene. In the subsequent hydrogenation process, the self-doping $\mathrm{Ti}^{3+}$ was introduced accompanied by the crystallization of amorphous $\mathrm{TiO}_{2}$. The narrowed bandgap caused by self-doping $\mathrm{Ti}^{3+}$ enhanced the visible light absorption and make the composites appear black. Both of them improved the photocatalytic performance of the synthesized black $\mathrm{TiO}_{2} /$ graphene composites. The band structure of the composite was analyzed by valence band XPS, revealing the reason for the high visible light catalytic performance of the composite. The results proved that the black $\mathrm{TiO}_{2} /$ graphene composites synthesized show attractive potential for applications in environmental and energy issues.

\section{Introduction}

Titanium dioxide $\left(\mathrm{TiO}_{2}\right)$ is regarded as an important photocatalyst because of its good photoelectric properties [1, 2]. Among the four crystal phases of $\mathrm{TiO}_{2}$, anatase and rutile phases are the most common and have been extensively researched due to their excellent photoactivity $[3,4]$. However, their large band gap (rutile at about $3.0 \mathrm{eV}$ and anatase at $3.2 \mathrm{eV}$ ) largely limits the activity to the ultraviolet (UV) region of the light. As photocatalysts, they only use less than $5 \%$ of the entire solar energy $[5,6]$. Moreover, the photogenerated electro-hole pairs in $\mathrm{TiO}_{2}$ will recombine during the conduction process instead of participating in the photocatalytic reaction. The recombination of electro-hole reduces the quantum efficiency, leading to a weaker photocatalytic efficiency [4]. Therefore, the absorption range of light and recombination of photogenerated electro-hole become the main factors of $\mathrm{TiO}_{2}$ photocatalysts.

In decades, significant attention has been attracted to improve the photocatalytic activity of $\mathrm{TiO}_{2}$. For example, through appropriate structural design, synthesis of metal and non-metal element doping and semiconductor composite materials [7-10]. In recent years, carbon nanomaterials have received great attention as composite materials, such as carbon nanotubes, graphene, etc. Especially graphene, because of its excellent electrical conductivity, chemical stability and adsorption capacity, is conducive to the improvement of $\mathrm{TiO}_{2}$ photocatalytic activity $[11,12]$. Many studies indicated that the excellent conductivity of graphene is conducive to the transfer of electrons. Therefore, the combination of $\mathrm{TiO}_{2}$ and graphene can significantly promote the recombination of photogenerated electron-hole, thereby improving photocatalytic performance $[13,14]$.

Huang et al. synthesized the $\mathrm{TiO}_{2} /$ graphene composites by a simple solvothermal method. The synthesized composites have excellent photocatalytic performance in degradation of formaldehyde and the effect of chemical bonds in the composites has been revealed [13]. Jung et al. reported a Graphene- 
2D mesoporous $\mathrm{TiO}_{2}$ synthesized via facile hydrothermal process, which showed outstanding degradation of methylene blue, due to the enhanced composite effects of graphene and $\mathrm{TiO}_{2}$ [14].

Although the $\mathrm{TiO}_{2}$-graphene composites greatly improve the catalytic performance of $\mathrm{TiO}_{2}$, this new composite material also has some problems, such as low visible light utilization [15, 16]. In 2011, Chen et al. presented a new method of synthesizing black $\mathrm{TiO}_{2}$ by hydrogenation to increase solar absorption [17]. Hydrogenation introduced disorder layers outside of the black $\mathrm{TiO}_{2}$ nanoparticles, dramatically changed the band gap of $\mathrm{TiO}_{2}$. The black $\mathrm{TiO}_{2}$ synthesized exhibit a long wavelength absorption and substantial visible light photocatalytic activities. These obviously changed properties make the appropriate integration of black $\mathrm{TiO}_{2}$ and graphene a promising method to develop the photocatalytic performance. Hence, it is necessary to synthesize and study the black $\mathrm{TiO}_{2} /$ graphene composites.

In this paper, a series of black $\mathrm{TiO}_{2} /$ graphene composites were synthesized by sol-gel method and subsequent hydrogenation process. Amorphous $\mathrm{TiO}_{2}$ is generated in situ on the surface of graphene by hydrolysis of organic titanium sources. Amorphous $\mathrm{TiO}_{2}$ precursor was used to synthesize black $\mathrm{TiO}_{2}$ by surface hydrogenation process. The synthesized photocatalyst had a narrow band gap, which can significantly extend the wavelength of light absorption. And the photocatalyst exhibited excellent photocatalytic performance due to the excellent photogenerated electro-hole separation ability of graphene. Moreover, the influence of graphene on the photocatalytic activity of black $\mathrm{TiO}_{2} /$ graphene composites has been systematically investigated by the degradation of methyl blue.

\section{Experimental}

\subsection{Synthesis of black $\mathrm{TiO}_{2} /$ graphene composites}

In typical, the black $\mathrm{TiO}_{2} /$ graphene composites were synthesized by a versatile sol-gel strategy as follows. Tetrabutyl titanate (TBOT) (CP, 98.0\%, Sinopharm Group Chemical Reagent Co., Ltd) and graphene (Strem Chemicals, Inc.) served as starting materials. First, a mixture solution of $5 \mathrm{~mL}$ TBOT and $250 \mathrm{~mL} \mathrm{C}{ }_{2} \mathrm{H}_{5} \mathrm{OH}$ (Eth) with different ratios of graphene were prepared. The polyethylene glycol (PEG) was used as surfactant to increase the surface binding between $\mathrm{TiO}_{2}$ and graphene. Then, the solution prepared was slowly dripped into the stirring solution of $250 \mathrm{~mL} \mathrm{C}_{2} \mathrm{H}_{5} \mathrm{OH}$ and $250 \mathrm{~mL} \mathrm{H}_{2} \mathrm{O}$. In the process, TBOT and the water in the solution occurred hydrolysis and polymerization to form a sol. The overall reaction process is $[18,19]$

$$
\mathrm{Ti}\left(\mathrm{OC}_{4} \mathrm{H}_{9}\right)_{4}+2 \mathrm{H}_{2} \mathrm{O} \rightarrow \mathrm{TiO}_{2}+4 \mathrm{C}_{4} \mathrm{H}_{9} \mathrm{OH} \text {. }
$$

After reaction for $1 \mathrm{~h}$ and precipitation for $3 \mathrm{~h}$, the resulting precipitate was centrifuged and washed 3 times with ethanol. Subsequently, the amorphous $\mathrm{TiO}_{2}$ graphene composites were obtained by drying at 
$80^{\circ} \mathrm{C}$ for $6 \mathrm{~h}$ and calcining at $200^{\circ} \mathrm{C}$ for $2 \mathrm{~h}$ in air. The obtained amorphous $\mathrm{TiO}_{2}$ graphene composites were gradually darkened in color with different graphene contents (1 wt $\%, 5 \mathrm{wt} \%, 10 \mathrm{wt} \%, 15 \mathrm{wt} \%)$.

Scheme 1 Schematic diagram showing synthetic procedure of black $\mathrm{TiO}_{2} /$ graphene composites

Finally, the amorphous $\mathrm{TiO}_{2}$ graphene composites were calcined in $\mathrm{H}_{2}$ flow at $500^{\circ} \mathrm{C}$ for $2 \mathrm{~h}$ under atmospheric pressure. Then the black $\mathrm{TiO}_{2}$ /graphene composites (denoted as BTG-1, BTG-5, BTG-10, BTG-15 correspond to different graphene content of $1 \mathrm{wt} \%, 5 \mathrm{wt} \%, 10 \mathrm{wt} \%, 15 \mathrm{wt} \%$ respectively) were synthesized. The process for synthesizing black $\mathrm{TiO}_{2} /$ graphene composite is shown in Scheme 1 .

\subsection{Characterization}

The morphology, structure and element distribution of the black $\mathrm{TiO}_{2} /$ graphene composites were examined by high-resolution transmission electron microscope (HRTEM, Tecnai G2 20). The crystal structure of the samples was detected by X-ray diffraction (XRD) on Rigaku D/MAX-2400. In order to confirm the chemical compositions and band status of the black $\mathrm{TiO}_{2} /$ graphene composites, XPS spectrum was characterized on AXIS-Ultra DLD-600W. The state of graphene was characterized by Raman spectroscopy with LabRAM HR800 using laser excitation at $532 \mathrm{~nm}$. The Raman spectroscopy scanning range is (100-3000 cm-1). In order to examine the light absorption range of the black $\mathrm{TiO}_{2}$ /graphene composites, UV-Vis absorption spectra were performed on a Shimadzu UV-3600 Plus UVVIS-NIR Spectrophotometer.

The photocatalytic activity of black $\mathrm{TiO}_{2} /$ graphene composites was determined by using them in the decomposition of the methyl blue (MB). The MB solution of $10 \mathrm{mg} / \mathrm{L}$ was prepared to test the visible light catalytic performance of the as-synthesized black $\mathrm{TiO}_{2} /$ graphene composites (BTG). $10 \mathrm{mg}$ of the BTG composites were added to $100 \mathrm{ml} \mathrm{MB}$ solution, stirred in the dark or 30 min to achieve adsorption/desorption equilibrium. The visible light irradiation of the photocatalysis experiment was from a $300 \mathrm{~W}$ halogen tungsten lamp with a cut-off filter $(\lambda>420 \mathrm{~nm})$. The temperature of the reaction solution is controlled at $20^{\circ} \mathrm{C}$ with a water-cooling system. In the photocatalysis experiment, $3 \mathrm{ml}$ of the reaction solution was taken every $10 \mathrm{~min}$, and the catalyst was removed by centrifugation (10000 rpm). The concentrations of residual MB were analyzed by the absorption band maximum $(660 \mathrm{~nm})$.

\section{Results And Discussion}

The XRD patterns of $\mathrm{TiO}_{2} /$ graphene composites before and after hydrogenation process are shown in Fig. $1(\mathrm{~A})$. The XRD patterns of the $\mathrm{TiO}_{2} /$ graphene composites before hydrogenation process show only some diffuse peaks, indicating that the $\mathrm{TiO}_{2}$ /graphene composites before hydrogenation are amorphous [20]. After the hydrogenation process, the peaks occur at $25.28^{\circ}(101), 37.80^{\circ}(004), 48.05^{\circ}(200), 53.89^{\circ}$ (105), $55.07^{\circ}(211), 62.69^{\circ}(204), 68.93^{\circ}(116), 70.31^{\circ}(220)$, and $75.03^{\circ}(215)$ (Fig. 1A(b)), corresponding to the diffractions of anatase $\mathrm{TiO}_{2}$ (JCPDS 21-1272) [18, 21]. It was indicated that the sample after 
surface hydrogenation is crystallized from amorphous to anatase structure, and the average crystal size is approximately $21 \mathrm{~nm}$ calculated by Scherrer formula, in agreement with TEM observation.

The structure of black $\mathrm{TiO}_{2} /$ graphene composites can also be characterized by Raman spectra. The Raman spectra of BTG with different graphene contents are shown in Fig. 1(B). The three bands at around $1365 \mathrm{~cm}^{-1}$ (D band), $1580 \mathrm{~cm}^{-1}$ ( $\mathrm{G}$ band) and $2700 \mathrm{~cm}^{-1}$ (2D band) are corresponding to graphene [22]. For all the BTG with different graphene contents, the Raman peaks occur at around $156 \mathrm{~cm}^{-1}\left(\mathrm{E}_{\mathrm{g}(1)}\right)$, $406 \mathrm{~cm}^{-1}\left(B_{1 \mathrm{~g}(1)}\right), 523 \mathrm{~cm}^{-1}\left(A_{1 \mathrm{~g}}+B_{1 \mathrm{~g}(2)}\right)$, and $646 \mathrm{~cm}^{-1}\left(E_{\mathrm{g}(2)}\right)$, which match with the characteristic peaks of anatase $\mathrm{TiO}_{2}$ [23]. Compared with the characteristic peaks of anatase $\mathrm{TiO}_{2}$, the $\mathrm{E}_{\mathrm{g}(1)}$ mode shift from $144 \mathrm{~cm}^{-1}$ of bare bulk $\mathrm{TiO}_{2}$ to $156 \mathrm{~cm}^{-1}$ of BTG synthesized. The shift toward high frequency indicates the ultra-dispersed characteristics of $\mathrm{TiO}_{2}$ nanoparticles and their combination with graphene [24]. And the disappearance of the graphene 2D band in $\mathrm{BTG}$ may be attributed to the composite of graphene and $\mathrm{TiO}_{2}$ [11]. From the Raman analysis, the characteristic peaks of $\mathrm{TiO}_{2}$ and graphene appeared in the spectra of $\mathrm{BTG}$, indicating that the black $\mathrm{TiO}_{2} /$ graphene composites were successfully synthesized.

Furthermore, the elemental composition of the black $\mathrm{TiO}_{2} /$ graphene composites was investigated by XPS. In Fig. 2(A), the characteristic peaks of $C 1 \mathrm{~s}, \mathrm{Ti} 2 \mathrm{p}$, and $\mathrm{O} 1 \mathrm{~s}$ are present at $284.6,457.8$, and 529.7 eV respectively. In the Ti $2 p$ XPS spectrum (Fig. 2(B)), the $\mathrm{Ti}_{2} 2 \mathrm{p}_{3 / 2}$ and $\mathrm{Ti}_{2} 2 \mathrm{p}_{1 / 2}$ of $\mathrm{TiO}_{2}$ are revealed at 457.8 and $463.3 \mathrm{eV}$. The Ti $2 \mathrm{p}_{3 / 2}$ peak of BTG shifts from $458.6 \mathrm{eV}$ to a lower binding energy corresponds to the presence of a high $\mathrm{Ti}^{3+}$ concentration [25-27]. All $\mathrm{Ti} 2 \mathrm{p}$ spectra are symmetrical on the low energy side, indicating that $\mathrm{TiO}_{2}$ is not doped with carbon [17]. The curve fit of $\mathrm{C} 1 \mathrm{~s}$ spectra of $\mathrm{BTG}$ is shown in Fig. 2(C). The peak at $284.5 \mathrm{eV}$ is ascribed to the $\mathrm{C}=\mathrm{C} / \mathrm{C}-\mathrm{C}$ bond, indicating the presence of graphene. The weak peak at 286.5 confirms the presence of the $\mathrm{C}-\mathrm{O}$ bond [28]. In addition, there is no Ti-C peak observed in both Fig. 2(B) and (C), which confirms that graphene does not exist as a dopant in the BTG composites. The curve fit of $O 1$ s spectra of BTG is shown in Fig. 2(D). The peak at 529.6 and $532.2 \mathrm{eV}$ are ascribed to Ti-O and $\mathrm{C}-\mathrm{OH}$ bonds. In both Fig. 2(C) and (D), the appearance of $\mathrm{C}-\mathrm{O}$ and $\mathrm{C}-\mathrm{OH}$ bonds indicate the existence of bond between carbon and oxygen atoms in the BTG composites. This phenomenon may be attributed to the oxidation of graphene since $\mathrm{TiO}_{2}$ is a well-known catalyst.

The morphology, structure and element distribution of the black $\mathrm{TiO}_{2} /$ graphene composites were examined by TEM. The TEM observation in Fig. $3(\mathrm{~A})$ shows that the $\mathrm{TiO}_{2}$ nanoparticles are well dispersed on the graphene sheets, and $10-50 \mathrm{~nm}$ in size.

The selected area electron diffraction (SAED) pattern is shown in the inset of Fig. 3(A). The (101), (004), (200), (204) and (105) diffraction rings are detected in the SAED pattern indicates that $\mathrm{TiO}_{2}$ in the composites is anatase structure [18]. The SAED result is consistent with the XRD characterization, which indicates that the $\mathrm{TiO}_{2}$ in the composite is anatase structure with excellent photocatalytic activity. Fig $3(\mathrm{~B})$ shows the HRTEM image. It can be seen that the size of individual $\mathrm{TiO}_{2}$ nanocrystal was approximately $15 \mathrm{~nm}$ in diameter. There was a disordered surface layer surrounding the $\mathrm{TiO}_{2}$ nanocrystal, 
as shown by the dotted red circle in Fig 3(B). The thickness of the disordered layer is about $1 \mathrm{~nm}$, which is consistent with the black $\mathrm{TiO}_{2}$ reported by Chen et al [17]. The disordered layer surrounding $\mathrm{TiO}_{2}$ nanocrystal is created by hydrogenation, which causes a significant color change and enhancement of visible light photocatalytic activity. The schematic diagram of sample color change (from blue to black) after hydrogenation is shown in Scheme 1. The inset in Fig 3(B) shows that the interplanar spacing is $3.58 \AA$, corresponding to the (101) plane of anatase $\mathrm{TiO}_{2}$. The Energy Dispersive X-ray (EDX) elemental mappings of $\mathrm{Ti}, \mathrm{C}, \mathrm{O}$ taken from the STEM image of Fig $3(\mathrm{C})$ are given in Fig $3(\mathrm{D}-\mathrm{F})$, respectively. It can be seen from the figures that the $\mathrm{Ti}$ and $\mathrm{O}$ elements are uniformly aggregated and dispersed on the $\mathrm{C}$ element of graphene, which is consistent with the TEM result of Fig. 3 (A). The results further demonstrate the successful assemblies of $\mathrm{TiO}_{2}$ on graphene in the black $\mathrm{TiO}_{2} /$ graphene composites.

Fig. 4(A) shows the UV-vis diffuse reflectance spectra (DRS) of the black $\mathrm{TiO}_{2} /$ graphene composites synthesized. The presence of graphene significantly improves the visible light absorption of the black $\mathrm{TiO}_{2} /$ graphene composites. The visible light absorption intensity of the black $\mathrm{TiO}_{2} /$ graphene composites is enhanced with increasing graphene content. In order to characterize the band gaps of the black $\mathrm{TiO}_{2}$ /graphene composites, the Kubelka-Munk function $\left(\mathrm{F}\left(R_{\infty}\right) \cdot \mathrm{E}\right)^{1 / \mathrm{n}}$ versus the energy of light $(\mathrm{E}=h v)$ is shown In Fig. 4(B) [29, 30]. For an indirect transition of anatase $\mathrm{TiO}_{2}, \mathrm{n}=2$ will give the best linear fit. As the graphene content increases from $1 \%$ to $15 \%$, the band gaps are estimated roughly to decrease from 3.05 to $2.94 \mathrm{eV}$. It is well known that the band gap energy of anatase $\mathrm{TiO}_{2}$ is $3.2 \mathrm{eV}$. The band gap around $3 \mathrm{eV}$ of black $\mathrm{TiO}_{2} /$ graphene composites is lower than that of anatase $\mathrm{TiO}_{2}$, which could be attributed to the self-doping of $\mathrm{Ti}^{3+}$. Moreover, the black $\mathrm{TiO}_{2} /$ graphene composites have enhanced light absorption in the range of visible light, which is consistent with the darker sample color with increasing graphene content. The results suggest that both graphene combination and self-doping of $\mathrm{Ti}^{3+}$ play a crucial role in the photocatalytic activity of the black $\mathrm{TiO}_{2} /$ graphene composites. The photocatalytic activity of the samples was shown in Fig. 4(C). Fig. 4(C) illustrates the normalized MB concentration in the degradation solution as a function of visible light irradiation time. After a visible light irradiation time of $60 \mathrm{~min}, 95 \%$, $98 \%, 99 \%$ and $96 \%$ MB are decomposed in the presence of the BTG-1, BTG-5, BTG-10 and BTG-15, respectively. A comparative experiment without catalyst during visible light irradiation exhibits only $15 \%$ MB decomposition. The photocatalytic process follows first-order kinetics, $c=c_{0} \exp (-k t)$, where $c_{0}$ and $c$ are the MB concentration before and after visible light irradiation, respectively [31]. The $k$ value in the formula represents the photocatalytic reaction rate. Through fitting calculation, the photocatalytic reaction rates $k$ for BTG-1, BTG-5, BTG-10 and BTG-15 are determined to be $2.88,3.61,3.99$ and $3.23 \mathrm{~h}^{-1}$, respectively. The BTG-10 exhibits the highest photocatalytic activity. To determine the recyclability of the black $\mathrm{TiO}_{2}$ /graphene composites, the BTG-10 is recycled under several visible light irradiation cycles. As shown in Fig. 4(D), after 5 cycles, the degradation rate of MB still reaches $90 \%$ in the presence of the BTG10 , indicating that the catalyst has good stability.

Since BTG-10 has the best catalytic activity in the $\mathrm{TiO}_{2}$ /graphene composites, $\mathrm{BTG}-10$ was selected for valence band (VB) XPS analysis in Fig. 5(A). The VB of BTG-10 is located at $2.68 \mathrm{eV}$, which is lower than 
the commonly used $\mathrm{TiO}_{2}(3.0 \mathrm{eV})$ [26]. The insets in Fig. 5(A) show the energy band diagrams. According to the energy band model, the conductance band (CB) can be calculated by $\mathrm{CB}=\mathrm{VB}-\mathrm{Eg}$, where Eg represents the energy of the band gaps. The Eg of BTG-10 is estimated to be $3.0 \mathrm{eV}$ by Fig. 4(B), so the $\mathrm{CB}$ of BTG-10 is calculated as $-0.32 \mathrm{eV}$. The results suggest that the disordered surface layer surrounding the $\mathrm{TiO}_{2}$ nanocrystal introduced by hydrogenation can upshift both the $\mathrm{VB}$ and $\mathrm{CB}$ edge of the $\mathrm{TiO}_{2} /$ graphene composites synthesized. According to the energy band analyses, the VB of the BTG-10 is higher than the $\mathrm{O}_{2} / \mathrm{H}_{2} \mathrm{O}$ and $\mathrm{CB}$ is $\mathrm{H}^{+} / \mathrm{H}_{2}$ potential, suggesting that the black $\mathrm{TiO}_{2} /$ graphene composites synthesized have attractive potential for applications in environmental and energy issues.

The photocatalytic mechanism is also proposed as shown in Fig. 5(B). The disordered surface layer introduced by hydrogenation narrows the band gap of the black $\mathrm{TiO}_{2} /$ graphene composites, which improves the optical absorption properties [32]. Consequently, the electrons in the VB can easily transition to the $\mathrm{CB}$ of $\mathrm{TiO}_{2}$ under visible light irradiation. It is well known that the graphene which nano-sized black $\mathrm{TiO}_{2}$ attached to has good electrical conductivity [33]. Therefore, electrons will be transferred to graphene instead of $\mathrm{CB}$, which is conducive to reducing the opportunities of electron-hole recombination and enhancing photocatalytic activity. However, the graphene itself has no photocatalytic activity, and excessive graphene will hinder the absorption of photons by $\mathrm{TiO}_{2}$. This hinder effect is the reason why BTG-10 has a higher catalytic activity than BTG-15.

\section{Conclusions}

A series of black $\mathrm{TiO}_{2} /$ graphene composites with different graphene contents were successfully synthesized. In the sol-gel process, $\mathrm{TiO}_{2}$ is generated in situ on the surface of graphene from TBOT as a titanium source. The good conductivity of graphene is beneficial to eliminating the opportunity of photogenerated electron-hole recombination. In the hydrogenation process, self-doping $\mathrm{Ti}^{3+}$ is introduced accompanied the crystallization of amorphous $\mathrm{TiO}_{2}$. The narrowed bandgap $(2.94 \sim 3.05 \mathrm{eV})$ caused by self-doping $\mathrm{Ti}^{3+}$ enhance the visible light absorption. Moreover, the nanostructured black $\mathrm{TiO}_{2}$-graphene hybrid materials show enhanced visible light photocatalytic activity in methyl blue degradation. The sample with $10 \mathrm{wt} \%$ graphene shows the highest photocatalytic activity and good stability. The incorporation of black $\mathrm{TiO}_{2}$ caused by hydrogenation and graphene composite expands the light absorption range and reduce the recombination of photogenerated electron-hole, both of which enhances the capacity of photodegrading organic dyes. Therefore, this work is expected to open up a new way for the synthesis of black $\mathrm{TiO}_{2} /$ graphene composites, and its high photocatalytic activity proves that the black $\mathrm{TiO}_{2}$ /graphene composites synthesized have attractive potential for applications in environmental and energy issues.

\section{Declarations}

\section{Acknowledgement}


This work was supported by the National Natural Science Foundation of China (No. 51805185), the Hubei Provincial technical innovation major program (No. 2017AAA109), the Wuhan Science and Technique Foundation (No. 2018010401011281), and Shenzhen Knowledge Innovation Program (No. 20180213102634650).

\section{References}

1. Hashimoto K, Irie H, Fujishima A (2005) Jpn J Appl Phys 44:8269

2. Fujishima A, K Honda (1972) nature: 37

3. Liu G, Yang HG, Pan J, Yang YQ, Lu GQ, Cheng HM (2014) Chem Rev 114:9559

4. Schneider J, Matsuoka M, Takeuchi M et al (2014) Chem Rev 114:9919

5. Lihong T, Xiaobo X, Chen (2017) Sci. Bull.: 431

6. Dette C, Pérezosorio MA, Kley CS et al (2014) Nano Lett 14:6533

7. Lee H-K, Lee S-W (2020) Mater Design 189:108497. Doi:10.1016/j.matdes.2020.108497

8. Faraldos M, Bahamonde A (2017) Catal Today 285:13

9. Yang HG, Sun $\mathrm{CH}$, Qiao SZ et al (2008) Nature 453:638

10. Bouslama M, Amamra MC, Jia Z et al (2012) ACS Catal 2:1884

11. Zhang XY, Li HP, Cui XL, Lin Y (2010) J Mater Chem 20:2801

12. Xie Q, Li J, Qiang T, Shi R (2012) J Mater Chem 22:13541

13. Huang Q, Tian S, Zeng D et al (2013) ACS Catal 3:1477

14. Cho KM, Kim KH, Choi HO, Jung HT (2015) Green Chem 17:3972

15. Zhang J, Zhu Z, Tang Y, Feng X (2013) J Mater Chem A 1:3752

16. Zhu P, Nair AS, Shengjie P, Shengyuan Y, Ramakrishna S (2012) ACS Appl Mater Interfaces 4:581

17. Chen X, Liu L, Yu PY, Mao SS (2011) Science 331:746. Doi:10.1126/science.1200448

18. Li Z, Zhu Y, Wang L, Wang J, Guo Q, Li J (2015) Appl Surf Sci 355:1051

19. Hague DC, Mayo MJ (1994) J. Am. Ceram. Soc. 77: 1957

20. Mansoorianfar M, Rahighi R, Hojjati-Najafabadi A, Mei C, Li D (2021) Mater Design 198:109314.

Doi:10.1016/j.matdes.2020.109314

21. Li W, Wang F, Feng S et al (2013) J Am Chem Soc 135:18300

22. Ferrari AC, Meyer JC, Scardaci V et al (2006) Phys Rev Lett 97:187401

23. Xing Z, Li J, Qiang W et al (2013) Eur. J. Inorg. Chem. 2013: 2411

24. Li N, Liu G, Zhen C, Li F, Zhang L, HM Cheng (2011) Adv Funct Mater 21:1717

25. Pan J, Sheng Y, Zhang J et al (2014) J Mater Chem A 2:18082

26. Zhou G, Shen L, Xing Z et al (2017) J Colloid Interface Sci 505:1031

27. Lu Z, Yip CT, Wang L, Huang H, Zhou L (2012) Chempluschem 77:991

28. Zhang Z, Xiao F, Guo Y, Wang S, Liu Y (2013) Appl Mater Interfaces 5:2227 
29. Scanlon DO, Dunnill CW, Buckeridge J et al (2013) Nat Mater 12:798

30. Reyes-Coronado D, Rodríguez-Gattorno G, Espinosa-Pesqueira ME, Cab C, De Coss R, G Oskam (2008) Nanotechnology 19:145605

31. Xiang Q, Yu J, Jaroniec M (2012) Chem Soc Rev 41:782

32. Ullattil SG, Periyat P (2016) J Mater Chem A 4:5854. Doi:10.1039/c6ta01993e

33. Xiong R, Hu K, AM Grant, et al (2016) Adv Mater 28:1501

\section{Figures}

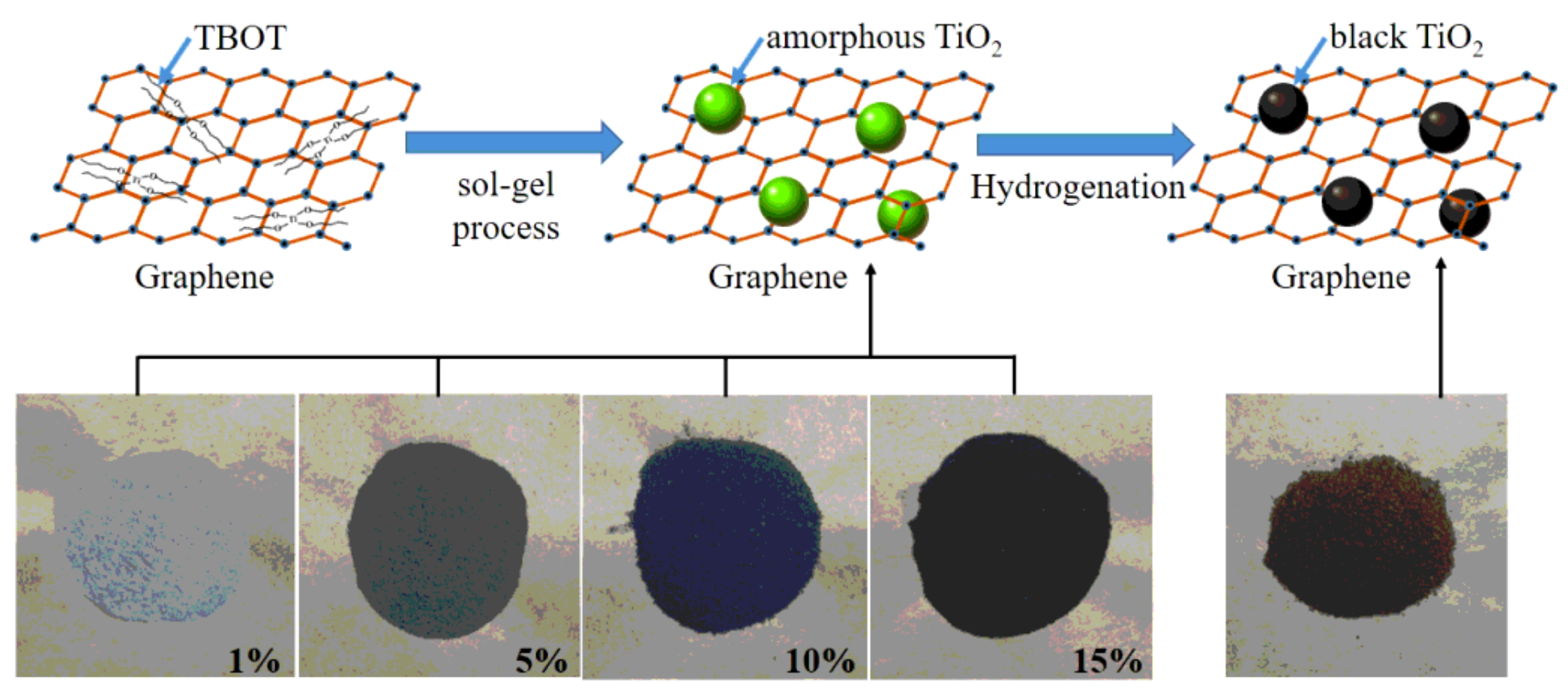

Figure 1

Schematic diagram showing synthetic procedure of black TiO2/graphene composites 


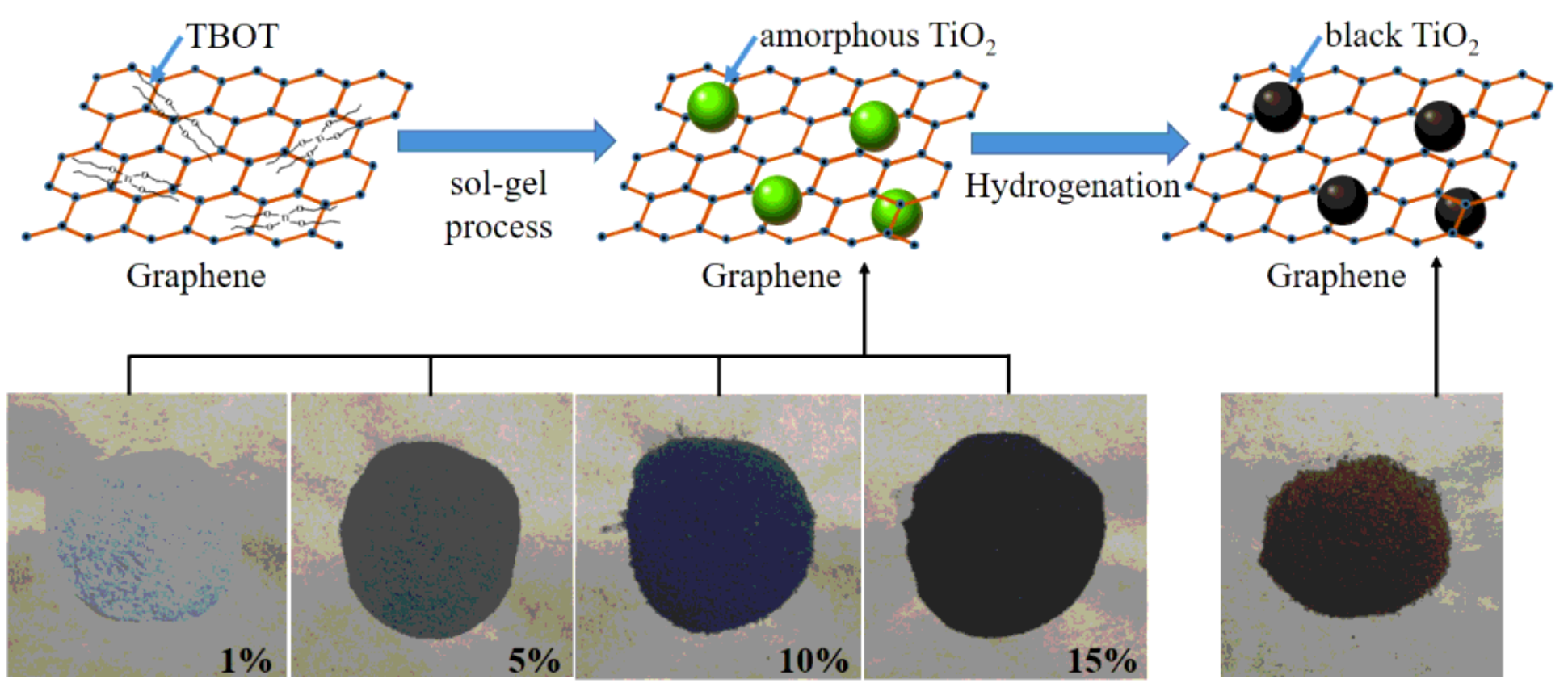

Figure 1

Schematic diagram showing synthetic procedure of black TiO2/graphene composites
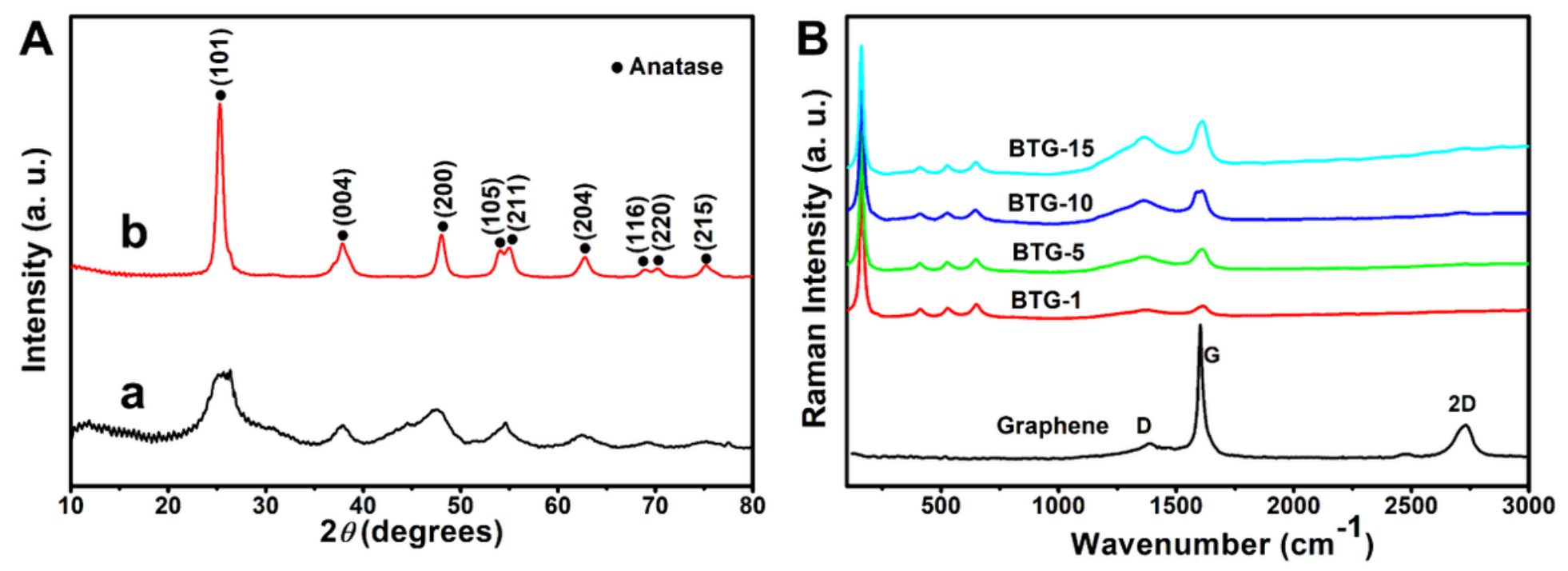

Figure 2

(A) XRD patterns of TiO2/graphene composites (a) before and (b) after hydrogenation process. (B) Raman spectra of black TiO2/graphene composites with different graphene contents. 

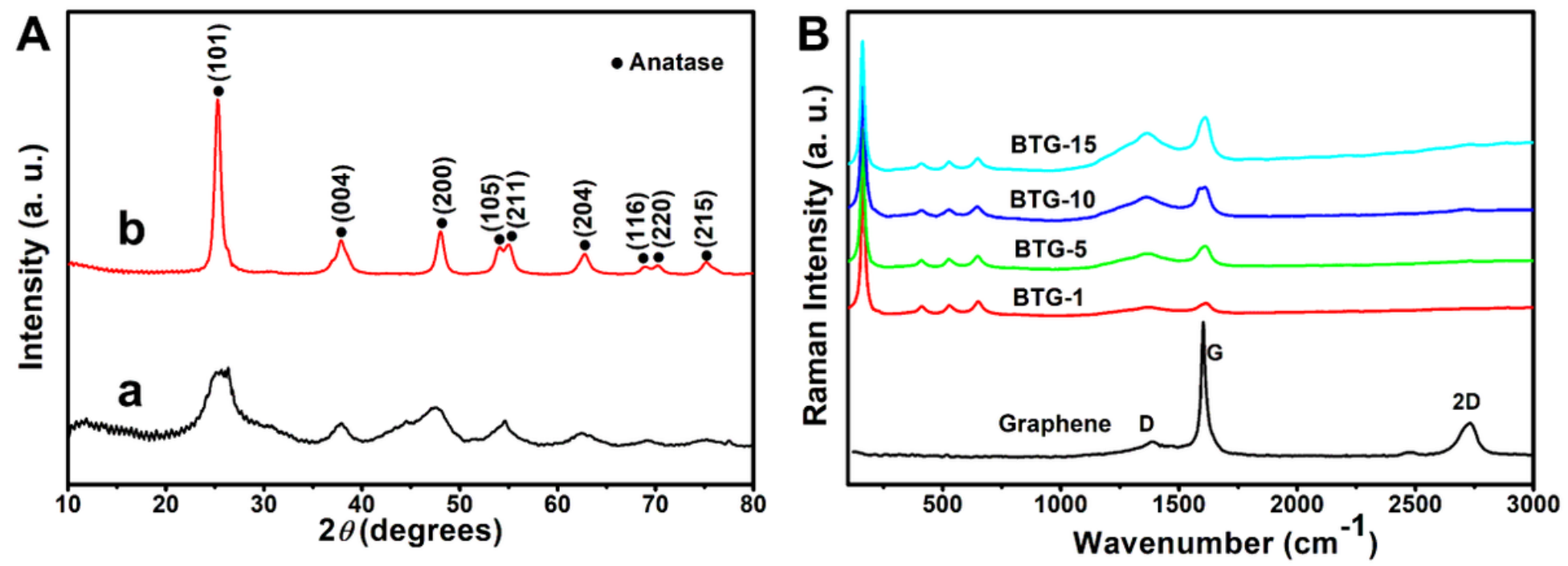

Figure 2

(A) XRD patterns of TiO2/graphene composites (a) before and (b) after hydrogenation process. (B) Raman spectra of black TiO2/graphene composites with different graphene contents.
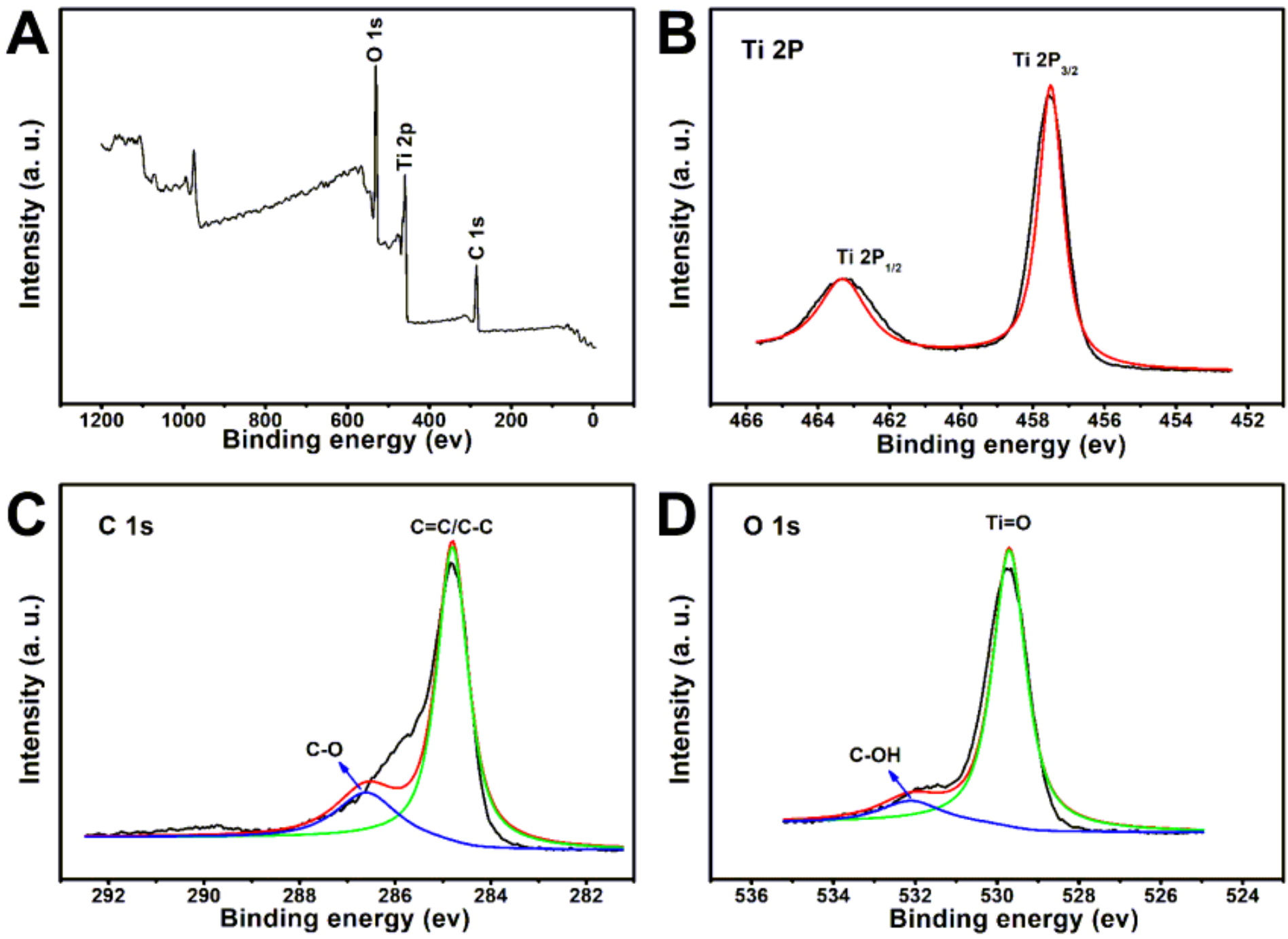
Figure 3

XPS spectra of black TiO2/graphene composites. (A) Full survey, (B) Ti 2p spectra. (C) C 1s spectra. (D) 0 1 s spectra.
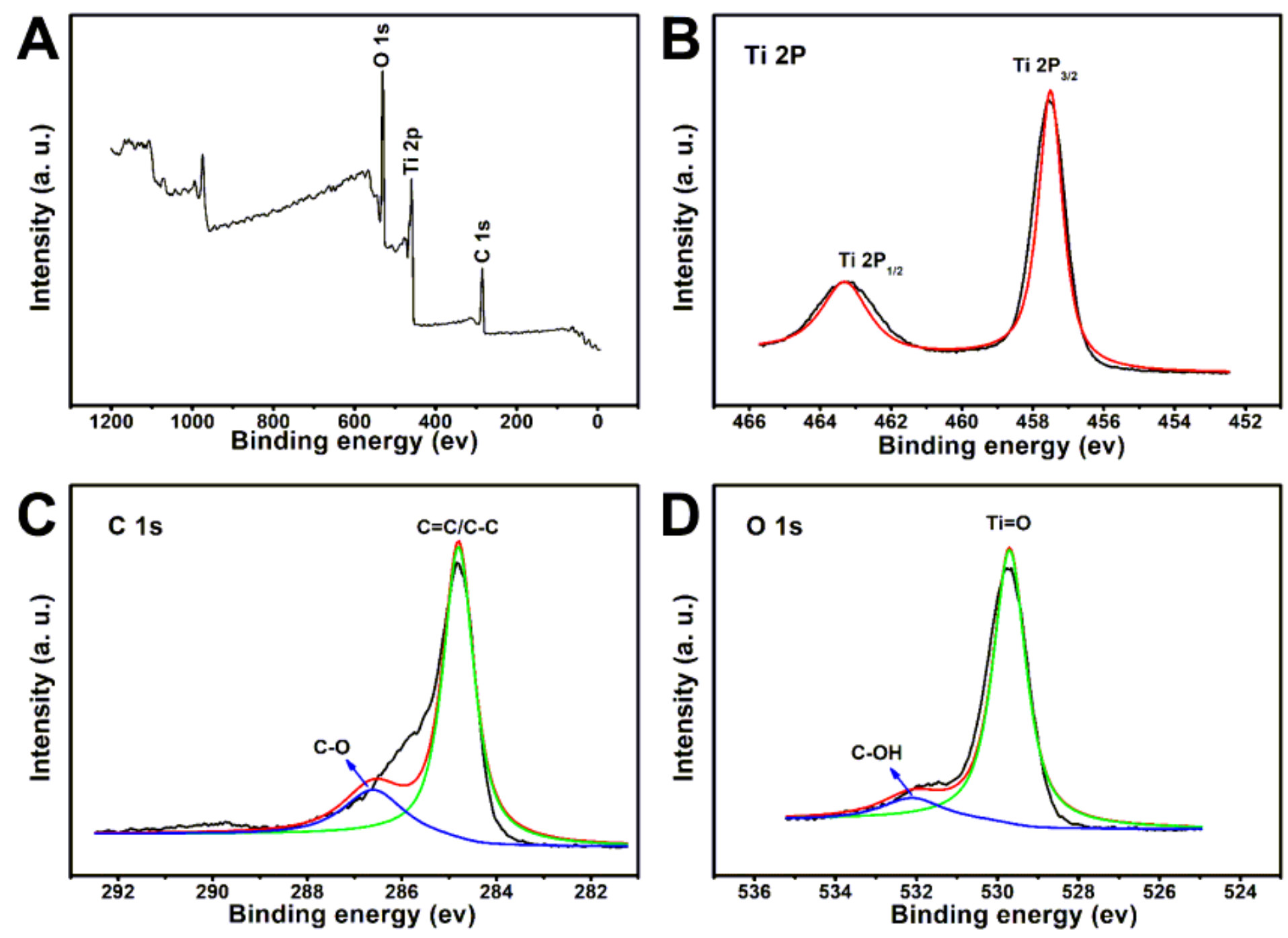

Figure 3

XPS spectra of black TiO2/graphene composites. (A) Full survey, (B) Ti 2p spectra. (C) C 1s spectra. (D) 0 1 s spectra. 


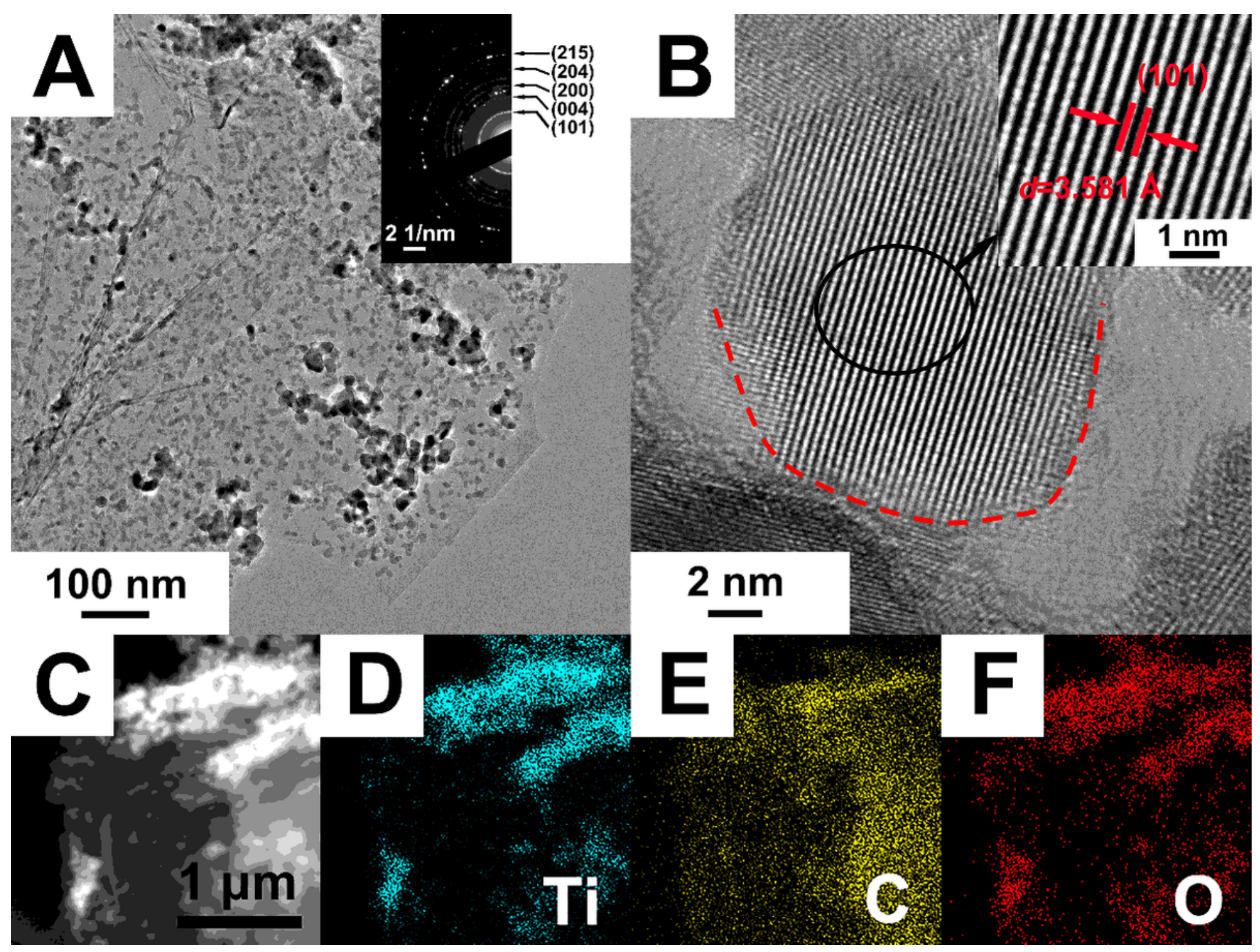

Figure 4

(A) TEM, (B) HRTEM and (C) STEM images of black TiO2/graphene composites. Elemental mapping of (D) titanium, (E) carbon and (F) oxygen taken from the whole area of (C). 


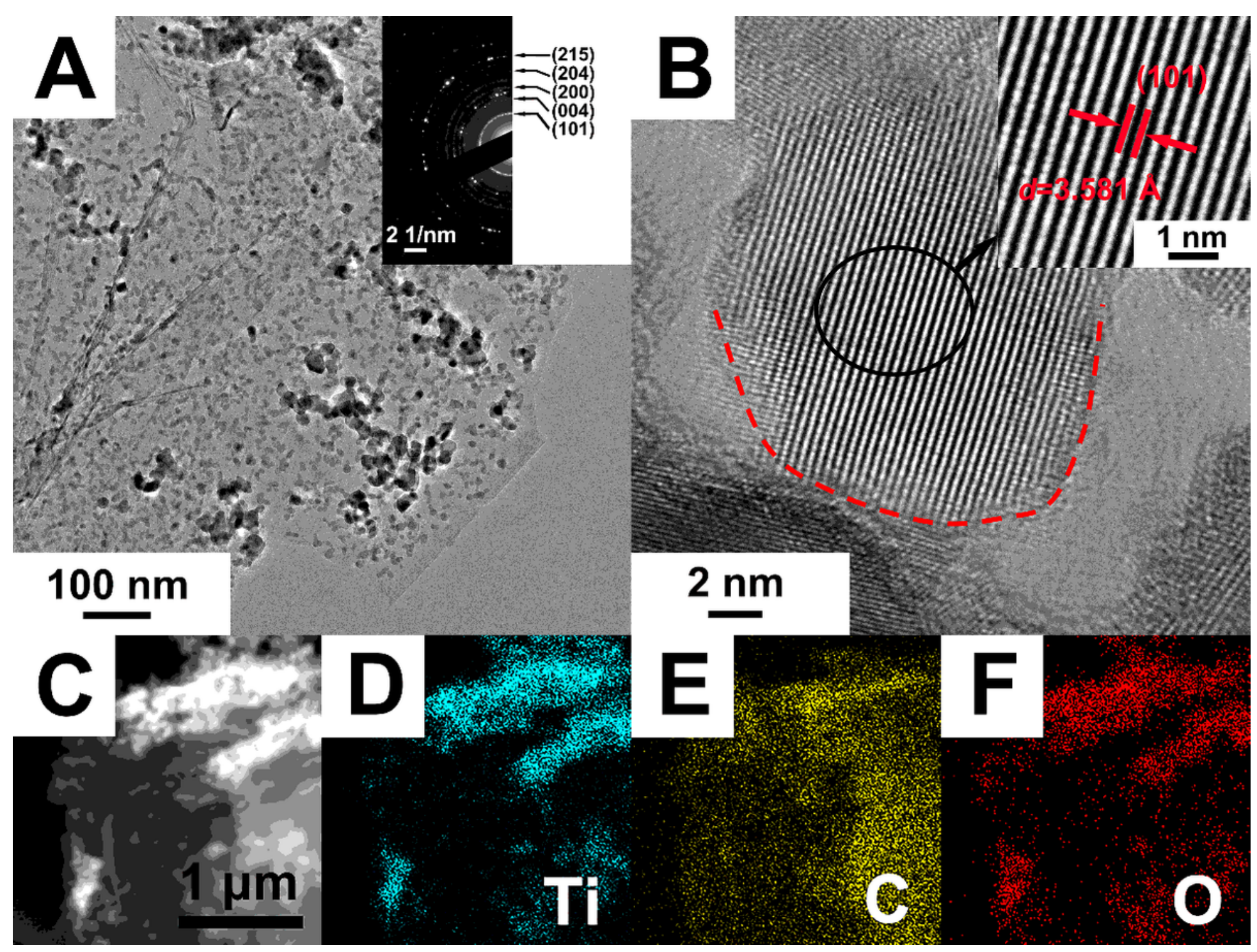

Figure 4

(A) TEM, (B) HRTEM and (C) STEM images of black TiO2/graphene composites. Elemental mapping of (D) titanium, (E) carbon and (F) oxygen taken from the whole area of (C). 

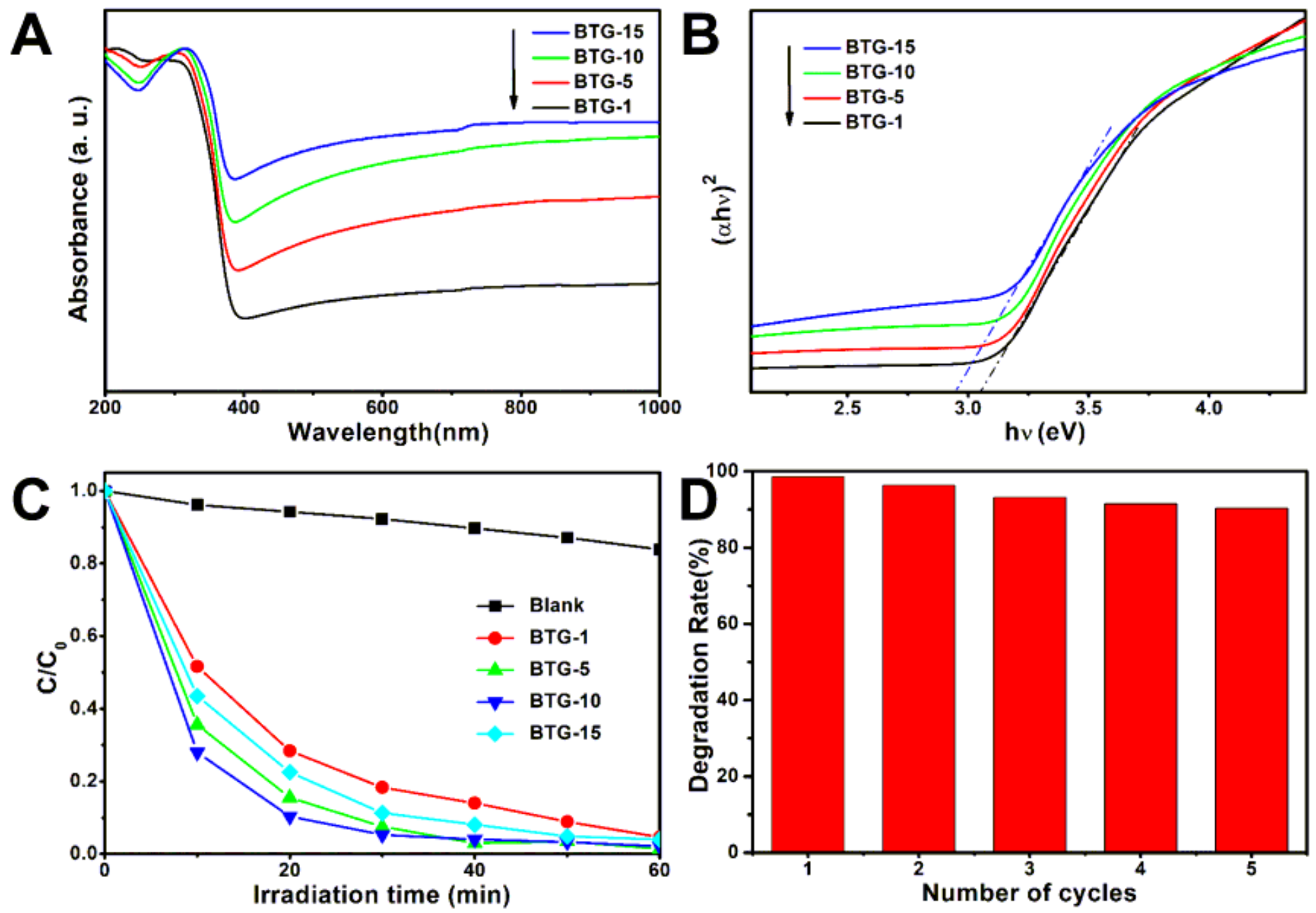

Figure 5

(A) UV-vis diffuse reflectance spectra of black TiO2/graphene composites, (B) the Kubelka-Munk function versus the energy of light, $(C)$ photocatalytic degradation of methylene blue (MB) under visible light $(\lambda \otimes$ $420 \mathrm{~nm})$, and (D) cycle test of the samples degradation MB. 

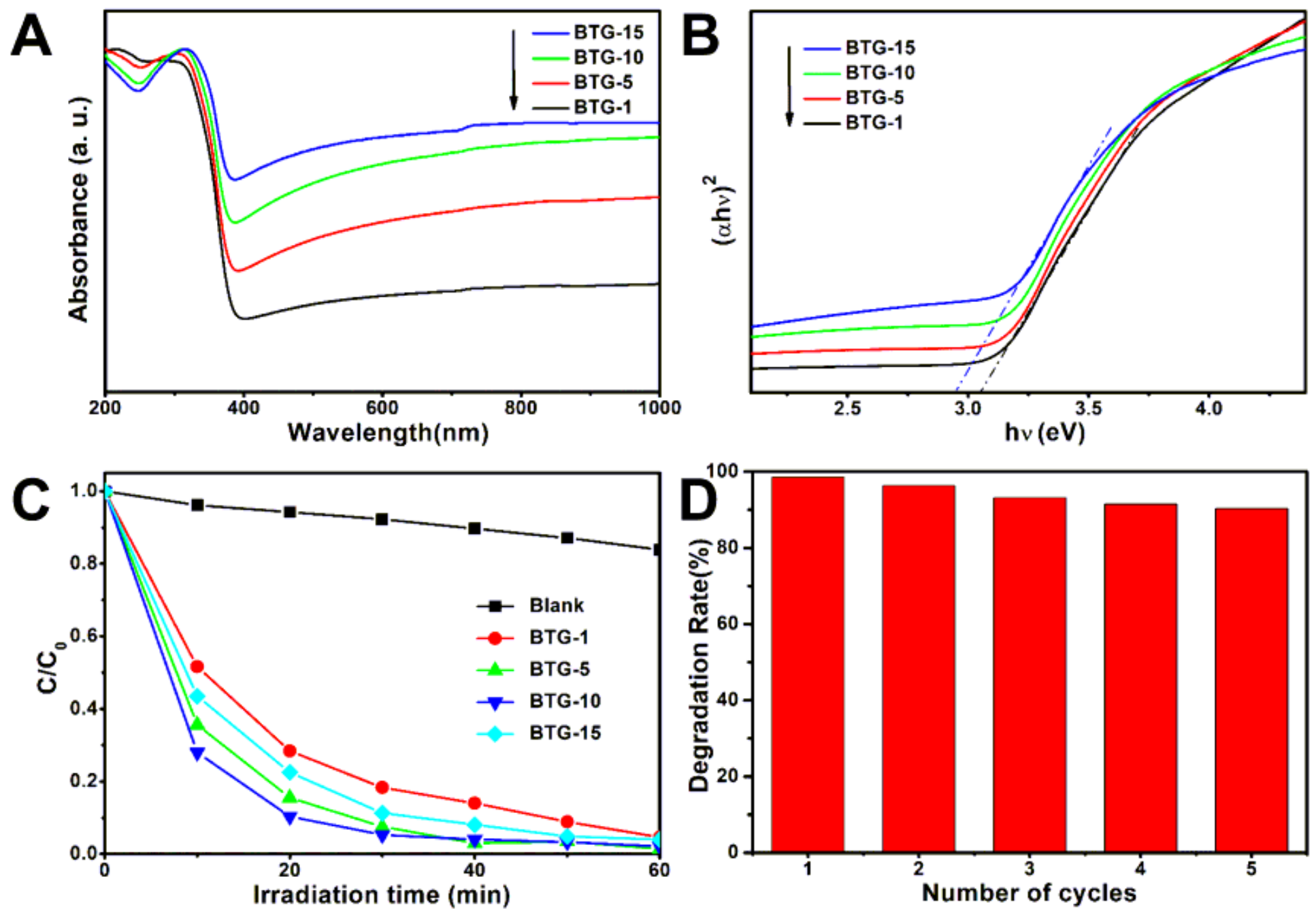

Figure 5

(A) UV-vis diffuse reflectance spectra of black TiO2/graphene composites, (B) the Kubelka-Munk function versus the energy of light, $(C)$ photocatalytic degradation of methylene blue (MB) under visible light $(\lambda \otimes$ $420 \mathrm{~nm}$ ), and (D) cycle test of the samples degradation MB.
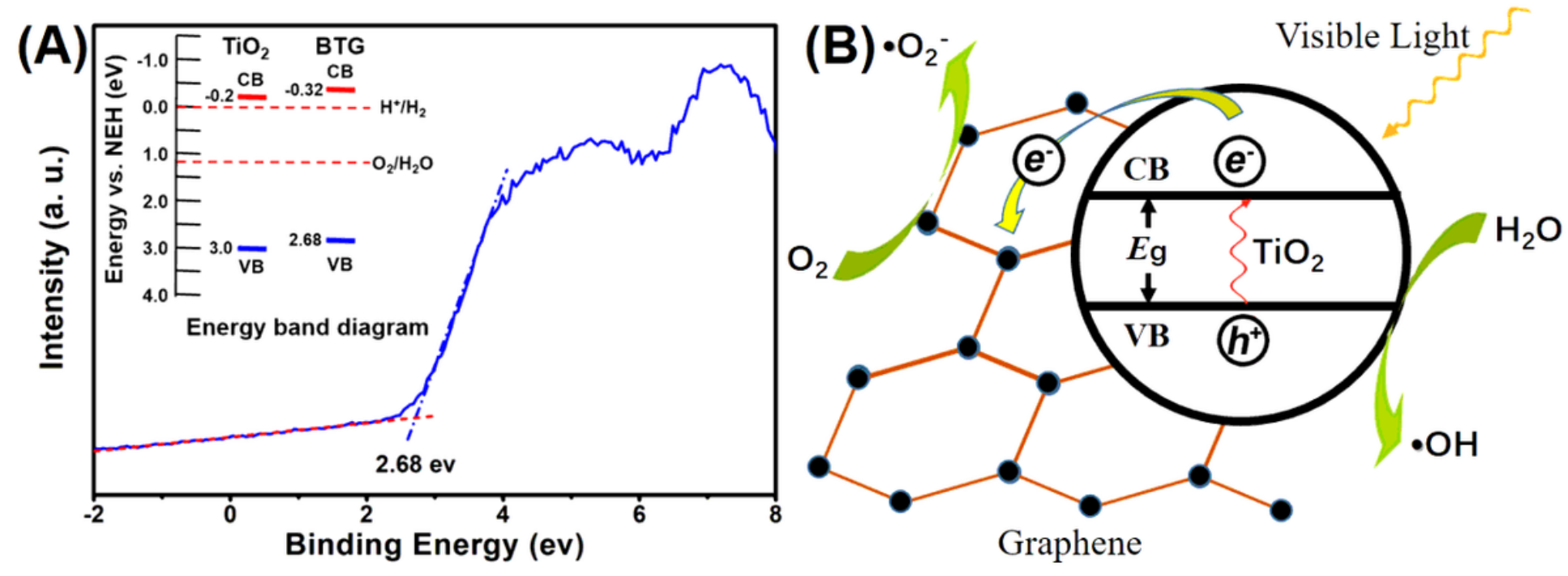
Figure 6

(A) The valence band XPS spectra of BTG-10, and (B) the proposed photocatalytic mechanism of BTG10.
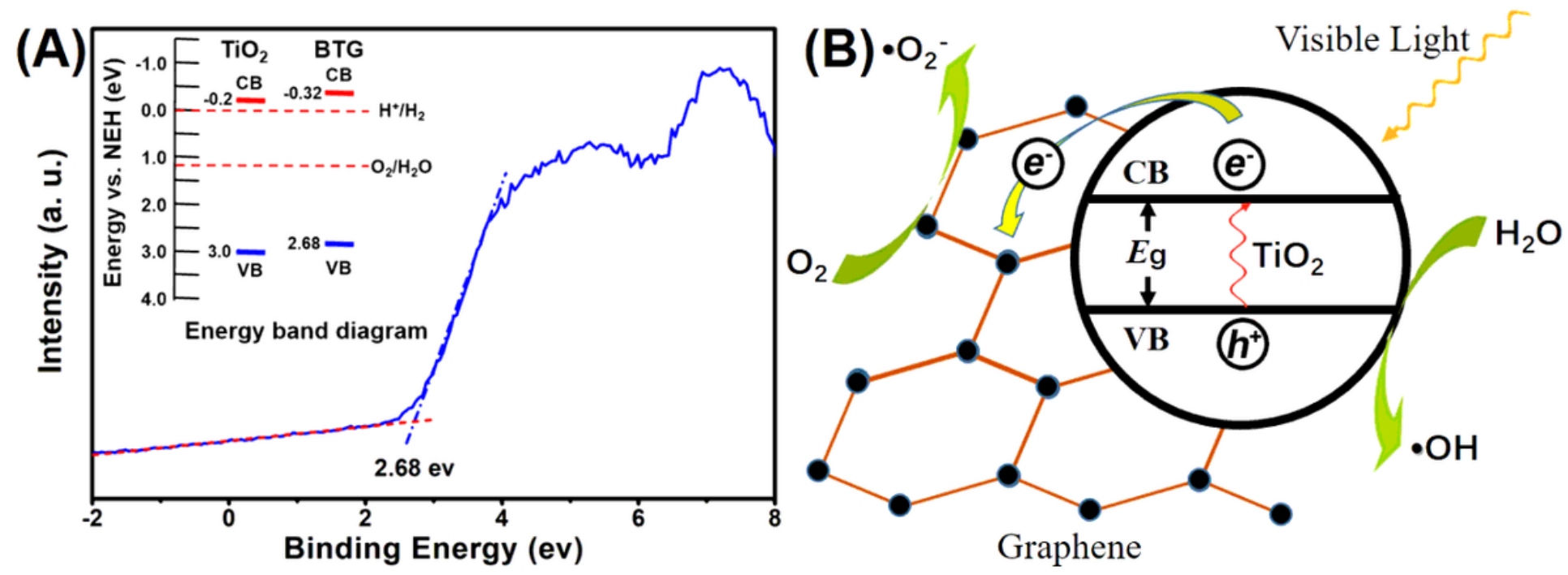

Figure 6

(A) The valence band XPS spectra of BTG-10, and (B) the proposed photocatalytic mechanism of BTG10. 